\title{
Investigation of non-lightlike tubular surfaces with Darboux frame in Minkowski 3-space
}

\author{
Emad Mohammed Solouma ${ }^{1,2}$ \\ ${ }^{1}$ Al Imam Mohammad Ibn Saud Islamic University, College of Science, Department of Mathematics and Statistics, Riyadh, KSA \\ ${ }^{2}$ Department of Mathematics, Faculty of Science, Beni-Suef University,Beni-Suef, Egypt
}

Received: 3 August 2016, Accepted: 26 September 2016

Published online: 9 October 2016.

\begin{abstract}
In this paper, the spacelike tubular surface with Darboux frame is introduced in Minkowski 3-space $\mathrm{E}_{1}^{3}$. Then, some characterizations were investigated for special curves on this tube with Darboux frame in Minkowski 3-space. Finally, we compute the Gaussian and mean curvature of tubular surface with Darboux frame.
\end{abstract}

Keywords: Minkowski space-time, tubular surface, Darboux frame, geodesic curvature.

\section{Introduction}

A canal surface is defined as envelope of a non-parameter set of spheres, centered at a spine curve $\alpha(t)$ with radius $r(t)$. When $r(t)$ is a constant function, the canal surface is the envelope of a moving sphere and is called a tube or tubular surface.

Canal surfaces are useful for representing long thin objects, e.g., pipes, poles, ropes, 3D fonts or intestines of body. Canal surfaces are also frequently used in solid and surface modelling for CAD/CAM. Representative examples are natural quadrics, torus, tubular surfaces and Dupin cyclides.

Canal surfaces have wide applications in CAGD, such as construction of blending surfaces, shape reconstruction, transition surfaces between pipes, robotic path planning, etc. (cf. [4,7,9]). Most of the literature on canal surfaces within the CAGD context has been motivated by the observation that canal surfaces with rational spine curve and rational radius function is rational, and it is therefore natural to ask for methods which allow one to construct a rational parameterizations of canal surfaces from its spine curve and radius function.

The canal surface can be considered as a generalization of the classical notion of an offset of a plane curve. In [2,3], the analysis and algebraic properties of offset curves are discussed in detail. In [1], Do Carmo discussed some geometrical features of pipe surfaces. Moreover, by using pipe surfaces, Do Carmo proved two very important theorems in Differential Geometry concerning the total curvature of space curves, namely Fenchel's theorem and Fary-Milnor theorem. In [5] Maekawa researched necessary and sufficient conditions for the regularity of tube (tubular) surfaces. Recently, Xu [11] are given a simple expression for the area and Gaussian curvature of canal surfaces. Also they proved that developable canal surface is either a cylinder or a cone. 
In this paper, we consider a spacelike tubular surface imbedded in Minkowski 3-space $\mathrm{E}_{1}^{3}$ with the spine spacelike center curve given with Darboux frame in Minkowski 3-space. This paper is organized as follows: Section 2 gives some basic concepts of the Frenet frame and Darboux frame of a curve in $\mathrm{E}_{1}^{3}$. Also this section provides formulas of the curvatures of surfaces in $\mathrm{E}_{1}^{3}$. Section 3 tells about the spacelike tubular surfaces in $\mathrm{E}_{1}^{3}$ with Darboux frame. We also obtain some characterizations for some special curves on these type of spacelike tubular surface.

\section{Preliminaries}

The Minkowski 3-space is three-dimensional Euclidean space provided with the Lorentzian inner product

$$
\langle u, v\rangle_{L}=-u_{1} v_{1}+u_{2} v_{2}+u_{3} v_{3}
$$

where $u=\left(u_{1}, u_{2}, u_{3}\right), v=\left(v_{1}, v_{2}, v_{3}\right)$ and denoted by $\mathrm{E}_{1}^{3}$. Any vector $u$ in $\mathrm{E}_{1}^{3}$ can be characterized as follows: the vector $\mathrm{u}$ is called spacelike, lightlike or timelike if $\langle u, u\rangle_{L}>0,\langle u, u\rangle_{L}=0$ or $\langle u, u\rangle_{L}<0$ respectively. The norm of a vector $u \in \mathrm{E}_{1}^{3}$ is given by $\|u\|=\sqrt{|\langle u, u\rangle|}$. Similarly, any arbitrary curve $\alpha=\alpha(s): I \rightarrow \mathrm{E}_{1}^{3}$ where $s$ is pseudo-arclength parameter, is called a spacelike curve if $\left\langle\alpha^{\prime}(s), \alpha^{\prime}(s)\right\rangle>0$, timelike if $\left\langle\alpha^{\prime}(s), \alpha^{\prime}(s)\right\rangle<0$ and null (lightlike) if $\left\langle\alpha^{\prime}(s), \alpha^{\prime}(s)\right\rangle=0$ and $\alpha^{\prime}(s) \neq 0$ for all $s \in I$.

The vectors $u=\left(u_{1}, u_{2}, u_{3}\right), v=\left(v_{1}, v_{2}, v_{3}\right) \in \mathrm{E}_{1}^{3}$ are orthogonal if and only if $\langle u, v\rangle=0$, and the Lorentzian cross product is given by

$$
\begin{aligned}
& u \wedge v=\left(u_{3} v_{2}-u_{2} v_{3}, u_{1} v_{3}-u_{3} v_{1}, u_{1} v_{2}-u_{2} v_{1}\right) \\
& e_{1} \wedge e_{2}=-e_{3}, e_{2} \wedge e_{3}=-e_{1}, e_{3} \wedge e_{1}=-e_{2} .
\end{aligned}
$$

Let $\alpha$ be any unit speed spacelike curve with timelike principal normal $N$ and spacelike binormal $B$ and with Frenet-Serret frame $\{t, n, b\}$, Frenet-Serret formulas of the curve $\alpha$ can be given as:

$$
\left[\begin{array}{l}
t^{\prime} \\
n^{\prime} \\
b^{\prime}
\end{array}\right]=\left[\begin{array}{ccc}
0 & \kappa(s) & 0 \\
\kappa(s) & 0 & \tau(s) \\
0 & \tau(s) & 0
\end{array}\right]\left[\begin{array}{l}
t \\
n \\
b
\end{array}\right]
$$

where $\langle t, t\rangle_{L}=\langle b, b\rangle_{L}=1,\langle n, n\rangle_{L}=-1$ and $\langle t, n\rangle_{L}=\langle n, b\rangle_{L}=\langle t, b\rangle_{L}=0$.

Definition 1.A surface $M$ in the Minkowski 3-space $\mathrm{E}_{1}^{3}$ is said to be spacelike, timelike surface if, respectively the induced metric on the surface is a positive definite Riemannian metric, Lorentz metric. In other words, the normal vector on the spacelike (timelike) surface is a timelike (spacelike) vector [6].

Let $\phi: U \subset \mathrm{E}^{2} \rightarrow \mathrm{E}_{1}^{3}, \phi(U)=M$ and $\gamma: I \subset \mathrm{R} \rightarrow U$ be a spacelike embedding and a regular curve, respectively. Then we have a curve $\alpha$ on the surface $M$ which is defined by $\alpha(s)=\phi(\gamma(s))$ and since $\phi$ is a spacelike embedding, we have a unit timelike normal vector field $\eta$ along the surface $M$ which is defined by $\eta=\frac{\phi_{x} \times \phi_{y}}{\left\|\phi_{x} \times \phi_{y}\right\|}$. Since $M$ is a spacelike surface,we can choose a future directed unit timelike normal vector field $\eta$ along the surface $M$. Hence we have a pseudoorthonormal frame $\{T, \eta, \zeta\}$ which is called the Lorentzian Darboux frame along the curve $\alpha$ where $\zeta(s)=T(s) \times \eta(s)$ is a unit spacelike vector. The corresponding Frenet formulae of $\alpha$ read

$$
\left[\begin{array}{l}
T^{\prime} \\
\eta^{\prime} \\
\zeta^{\prime}
\end{array}\right]=\left[\begin{array}{ccc}
0 & \kappa_{n} & \kappa_{g} \\
\kappa_{n} & 0 & \tau_{g} \\
-\kappa_{g} & \tau_{g} & 0
\end{array}\right]\left[\begin{array}{l}
T \\
\eta \\
\zeta
\end{array}\right]
$$


where $\kappa_{n}(s)=-\left\langle T^{\prime}(s), \eta(s)\right\rangle, \kappa_{g}(s)=\left\langle T^{\prime}(s), \zeta(s)\right\rangle$ and $\tau_{g}(s)=-\left\langle\zeta^{\prime}(s), \eta(s)\right\rangle$ are geodesic curvature, the asymptotic curvature, and the principal curvature of $\alpha$ on the surface $M$ in $\mathrm{E}_{1}^{3}$ respectively, and $s$ is arc-length parameter of $\alpha$. In particular, the following relations hold:

$$
\begin{aligned}
& \langle T, T\rangle=\langle\zeta, \zeta\rangle=1, \quad\langle\eta, \eta\rangle=-1 \\
& \langle T, \eta\rangle=\langle\eta, \zeta\rangle=\langle\zeta, T\rangle=0 \\
& T \wedge \eta=\zeta, \eta \wedge \zeta=-T, \zeta \wedge T=\eta
\end{aligned}
$$

Both $\kappa_{n}$ and $\kappa_{g}$ may be positive or negative. Specifically, $\kappa_{n}$ is positive if $\alpha$ curves towards the normal vector $\eta$, and $\kappa_{g}$ is positive if $\alpha$ curves towards the tangent normal vector $\xi$.

Let $\left.X_{(} u, v\right)$ be a local parametrization of $M$. The tangent vectors to the parametric curves of the surface $M$ are

$$
X_{u}=\frac{\partial X}{\partial u}, \quad X_{v}=\frac{\partial X}{\partial v} .
$$

The unite normal vector field on $M$ is given by

$$
N=\frac{X_{u} \wedge X_{v}}{\left|X_{u} \wedge X_{v}\right|}
$$

where $\wedge$ means the cross product in $E_{1}^{3}$. The first fundamental coefficients are given by

$$
g_{11}=\left\langle X_{u}, X_{u}\right\rangle, \quad g_{12}=\left\langle X_{u}, X_{v}\right\rangle, \quad g_{22}=\left\langle X_{v}, X_{v}\right\rangle
$$

The second fundamental coefficients

$$
h_{11}=\left\langle N, X_{u u}\right\rangle, \quad h_{12}=\left\langle N, X_{u v}\right\rangle \quad h_{22}=\left\langle N, X_{v v}\right\rangle
$$

The Gaussian and mean curvature are given by [6]

$$
K=\frac{h_{11} h_{22}-h_{12}^{2}}{g_{11} g_{22}-g_{12}^{2}}, \quad H=\frac{h_{11} g_{22}-2 h_{12} g_{12}+h_{22} g_{11}}{2\left(g_{11} g_{22}-g_{12}^{2}\right)} .
$$

Let the center curve $c(u)$ be on the surface $M$. Since the characteristic circles of canal surface lie in the plane which is perpendicular to the tangent of center curve $c(u)$, we can write spacelike tubular surface with Darboux frame in the Minkowski 3-space $\mathrm{E}_{1}^{3}$ as

$$
X(u, v)=c(u)+r(\zeta(u) \cosh v+\eta(u) \sinh v),
$$

where $\eta$ is the unit normal of the surface $M$ along the curve $c(u)$.

\section{Characterization of special curves on spacelike tubular surface with Darboux frame}

In this section, we investigate the relation between parameter curves and special curves such as geodesics, asymptotic curves and lines of curvature on the spacelike tubular surface given by Eqn. (7).

Theorem 1.For the regular spacelike tubular surface with Darboux frame in $\mathrm{E}_{1}^{3}$ and given by Eqn. (7).

(i) v-parameter curves are geodesics, 
(ii) $u$-parameter curves are geodesics if and only if $\kappa_{g}, \kappa_{n}$ and $\tau_{g}$ satisfy the following conditions

$$
\begin{aligned}
& \kappa_{n} \cosh v-\kappa_{g} \sinh v-r \kappa_{n} \kappa_{g} \cosh 2 v+\frac{1}{2} r\left(\kappa_{n}^{2}+\kappa_{g}^{2}\right) \sinh 2 v+r \tau_{g}^{\prime}=0, \\
& \left(\kappa_{g}^{\prime}-\kappa_{n} \tau_{g}\right) \cosh v-\left(\kappa_{n}^{\prime}-\kappa_{g} \tau_{g}\right) \sinh v=0 .
\end{aligned}
$$

Proof. The tangent space to the spacelike tubular surface $M$ given by Eqn. (7) at an arbitrary point $p$ of $M$ is spanned by

$$
\begin{aligned}
& X_{u}=\left(1-r \kappa_{g} \cosh v+r \kappa_{n} \sinh v\right) T+\left(r \tau_{g} \cosh v\right) \eta+\left(r \tau_{g} \sinh v\right) \zeta \\
& X_{v}=(r \cosh v) \eta+(r \sinh v) \zeta
\end{aligned}
$$

Hence the second partial derivatives of the parametric curves are given as follows:

$$
\left\{\begin{aligned}
X_{u u}= & \left(r \kappa_{n}^{\prime} \sinh v-r \kappa_{g}^{\prime} \cosh v-r \kappa_{g} \tau_{g} \sinh v+r \kappa_{n} \tau_{g} \cosh v\right) T \\
& +\left(\kappa_{n}+r \kappa_{n}^{2} \sinh v-r \kappa_{n} \kappa_{g} \cosh v+r \tau_{g}^{\prime} \cosh v+r \tau_{g}^{2} \sinh v\right) \eta \\
& +\left(\kappa_{g}-r \kappa_{g}^{2} \cosh v+r \kappa_{n} \kappa_{g} \sinh v+r \tau_{g}^{\prime} \sinh v+r \tau_{g}^{2} \cosh v\right) \zeta \\
X_{u v}= & \left(r \kappa_{n} \cosh v-r \kappa_{g} \sinh v\right) T+\left(r \tau_{g} \sinh v\right) \eta+\left(r \tau_{g} \cosh v\right) \zeta \\
X_{v v}= & (r \sinh v) \eta+(r \cosh v) \zeta .
\end{aligned}\right.
$$

Thereafter, from equations (3), (9) and (10) we obtain

$$
N=-(\sinh v) \eta-(\cosh v) \zeta
$$

and

$$
\begin{gathered}
\omega=N \wedge X_{v v}=(r \sinh v \cosh v) T-(r \sinh v \cosh v) T=0, \\
\lambda=N \wedge X_{u u}=-\left(\kappa_{n} \cosh v-r \kappa_{n} \kappa_{g} \cosh 2 v-\kappa_{g} \sinh v+\frac{1}{2} r\left(\kappa_{n}^{2}+\kappa_{g}^{2}\right) \sinh 2 v+r \tau_{g}^{\prime}\right) T \\
+r \cosh v\left(\kappa_{n}^{\prime} \sinh v-\kappa_{g}^{\prime} \cosh v-\kappa_{g} \tau_{g} \sinh v+\kappa_{n} \tau_{g} \sin v \cosh v\right) \eta \\
-r \sinh v\left(\kappa_{n}^{\prime} \sinh v-\kappa_{g}^{\prime} \cosh v-\kappa_{g} \tau_{g} \sinh v+\kappa_{n} \tau_{g} \sin v \cosh v\right) \zeta .
\end{gathered}
$$

Since $\omega=0$, this imply that $v$-parameter curves are geodesics. Also, because $T, \zeta$ and $\eta$ are linearly independent, this means that $\lambda=0$ if and only if

$$
\left\{\begin{array}{l}
\kappa_{n} \cosh v-r \kappa_{n} \kappa_{g} \cosh 2 v-\kappa_{g} \sinh v+\frac{1}{2} r\left(\kappa_{n}^{2}+\kappa_{g}^{2}\right) \sinh 2 v+r \tau_{g}^{\prime}=0 \\
r \cosh v\left(\kappa_{n}^{\prime} \sinh v-\kappa_{g}^{\prime} \cosh v-\kappa_{g} \tau_{g} \sinh v+\kappa_{n} \tau_{g} \sin v \cosh v\right) \\
-r \sinh v\left(\kappa_{n}^{\prime} \sinh v-\kappa_{g}^{\prime} \cosh v-\kappa_{g} \tau_{g} \sinh v+\kappa_{n} \tau_{g} \sin v \cosh v\right)=0
\end{array}\right.
$$

Thus from the last two equations, we have

$$
\left(\kappa_{g}^{\prime}-\kappa_{n} \tau_{g}\right) \cosh v-\left(\kappa_{n}^{\prime}-\kappa_{g} \tau_{g}\right) \sinh v=0 .
$$

Then $\kappa_{g}, \kappa_{n}$ and $\tau_{g}$ hold the Eqn. (8).

As a consequence of the above theorem we obtain the following result; 
Corollary 1. Let $c(u)$ be a spacelike geodesic on the surface $M$ with Darboux frame in Minkowski 3-space $\mathrm{E}_{1}^{3}$. If $u$ parameter curves are also geodesics on $X(u, v)$, then the curvatures $\kappa$ and $\tau$ of $c(u)$ satisfy the following equation

$$
\kappa(r \kappa \sinh v+2) \sinh v-r \tau^{2}=\mu
$$

where $\mu$ is a constant.

Proof. Since the center curve $c(u)$ is a geodesic, then $k_{g}=0, \kappa_{n}=\kappa$ and $\tau_{g}=\tau$. From Eqn. (8), we get

$$
\begin{aligned}
& \kappa(r \kappa \sinh v+1) \cosh v+r \tau^{\prime}=0, \\
& -\kappa^{\prime} \sinh v-\kappa \tau \cosh v=0 .
\end{aligned}
$$

Eliminating $\cosh v$ from the previous two equations, we obtain

$$
r \kappa \kappa^{\prime} \sinh ^{2} v+\kappa^{\prime} \sinh v-r \tau \tau^{\prime}=0 .
$$

By integrating Eqn. (12), it follows that

$$
r \kappa^{2} \sinh ^{2} v+2 \kappa \sinh v-r \tau^{2}=\mu
$$

Corollary 2. Let the center curve $c(u)$ be an asymptotic spacelike curve on the surface $M$ with Darboux frame in Minkowski 3-space $\mathrm{E}_{1}^{3}$. If $u$-parameter curves are also asymptotic curves on $X(u, v)$, then the curvatures $\kappa$ and $\tau$ of $c(u)$ satisfy the following equation

$$
\kappa(r \kappa \cosh v-2) \cosh v+r \tau^{2}=\mu
$$

where $\mu$ is a constant.

Proof. Since the center curve $c(u)$ is an asymptotic curve on $M$, then $k_{n}=0, \kappa_{g}=\kappa$ and $\tau_{g}=\tau$. So, from Eqn. (8), we get

$$
\begin{aligned}
& \kappa(r \kappa \cosh v-1) \sinh v+r \tau^{\prime}=0, \\
& \kappa^{\prime} \cosh v-\kappa \tau \sinh v=0 .
\end{aligned}
$$

Eliminating $\sinh v$ from the two previous equations, we obtain

$$
r \kappa \kappa^{\prime} \cosh ^{2} v-\kappa^{\prime} \cosh v+r \tau \tau^{\prime}=0 .
$$

By integrating Eqn. (13), it follows that

$$
r \kappa^{2} \cosh ^{2} v-2 \kappa \cosh v+r \tau^{2}=\mu
$$

Theorem 2. For the regular spacelike tubular surface with Darboux frame in $E_{1}^{3}$ and given by Eqn. (7).

(i) v-parameter curves cannot be asymptotic curves,

(ii) $u$-parameter curves are asymptotic curves if and only if $X(u, v)$ is generated by a moving sphere with the radius function

$$
r=\frac{\kappa_{n} \sinh v-\kappa_{g} \cosh v}{\tau_{g}^{2}-\left(\kappa_{n} \sinh v-\kappa_{g} \cosh v\right)^{2}}=\mu
$$

such that $\mu$ is constant 
Proof. From equations (5), (10) and (11), we get

$$
\begin{aligned}
& h_{22}=\left\langle N, X_{v v}\right\rangle=-r \cosh ^{2} v+r \sinh ^{2} v=-r \neq 0, \\
& h_{11}=\left\langle N, X_{u u}\right\rangle=\left(\kappa_{n} \sinh v-\kappa_{g} \cosh v\right)\left[1+r\left(\kappa_{n} \sinh v-\kappa_{g} \cosh v\right)\right]-r \tau_{g}^{2} .
\end{aligned}
$$

Then $h_{22} \neq 0$, implies that $v$-parameter curves cannot be asymptotic curves. Also, since $u$-parameter curves are asymptotic curves, then $h_{11}=0$, which leads to

$$
r=\frac{\kappa_{n} \sinh v-\kappa_{g} \cosh v}{\tau_{g}^{2}-\left(\kappa_{n} \sinh v-\kappa_{g} \cosh v\right)^{2}}=\mu
$$

such that $\mu$ is constant.

Corollary 3. Let $u$-parameter curves be asymptotic curves on the spacelike tubular surface $X(u, v)$ with Darboux frame in $\mathrm{E}_{1}^{3}$ and given by Eqn. (7).

(i) If the center curve $c(u)$ is a geodesic on the surface $M$, then

$$
r=\frac{\kappa \sinh v}{\tau^{2}-\kappa^{2} \sinh ^{2} v}=\mu
$$

(ii) If the center curve $c(u)$ is an asymptotic curve on the surface $M$, then

$$
r=\frac{\kappa \cosh v}{\kappa^{2} \cosh ^{2} v-\tau^{2}}=\mu
$$

(iii) If the center curve $c(u)$ is a line of curvature on the surface $M$, then

$$
r=\frac{1}{\kappa_{g} \cosh v-\kappa_{n} \sinh v}=\mu .
$$

Proof. From Theorem 2, since $u$-parameter curves are asymptotic curve, then from Eqn. (14) we have

$$
r=\frac{\kappa_{n} \sinh v-\kappa_{g} \cosh v}{\tau_{g}^{2}-\left(\kappa_{n} \sinh v-\kappa_{g} \cosh v\right)^{2}}=\mu
$$

(i) Since the center curve $c(u)$ is a geodesic on the surface $M$. So, $\kappa_{g}=0, \kappa_{n}=\kappa$ and $\tau_{g}=\tau$. If we replace these in Eqn. (14) we get

$$
r=\frac{\kappa \sinh v}{\tau^{2}-\kappa^{2} \sinh ^{2} v}=\mu
$$

(ii) Since the center curve $c(u)$ is an asymptotic curve on the surface $M$, then $\kappa_{n}=0, \kappa_{g}=\kappa$ and $\tau_{g}=\tau$. If we replace these in Eqn. (14) we get

$$
r=\frac{\kappa \cosh v}{\kappa^{2} \cosh ^{2} v-\tau^{2}}=\mu
$$

If we take for example $v=0$, it follows that $r=\frac{\kappa}{\kappa^{2}-\tau^{2}}$ is constant and therefore the center curve $c(u)$ become Mannheim curve. In this situation, while $c(u)$ is a Mannheim curve, the $u$-parameter curve $v=0 ; X(u, 0)=c(u)+$ $r \zeta(u)$ is an asymptotic curve on the tubular $X(u, v)$.

(iii) Since the center curve $c(u)$ is a line of curvature on the surface $M$, then $\tau_{g}=0$. If we put this in Eqn. (14) we get

$$
r=\frac{1}{\kappa_{g} \cosh v-\kappa_{n} \sinh v}=\mu .
$$


Theorem 3. The parameter curves of spacelike tubular surface $X(u, v)$ with Darboux frame in Minkowski 3-space $\mathrm{E}_{1}^{3}$ are lines of curvature if and only if the center curve $c(u)$ is a line of curvature on the surface $M$.

Proof. From equations (5), (9) and (10) we obtain

$$
\begin{aligned}
& g_{12}=\left\langle X_{u}, X_{v}\right\rangle=r^{2} \tau_{g} \sinh ^{2} v-r^{2} \tau_{g} \cosh ^{2} v=-r^{2} \tau_{g}, \\
& h_{12}=\left\langle N, X_{u v}\right\rangle=-r \tau_{g} \cosh ^{2} v+r \tau_{g} \sinh ^{2} v=-r \tau_{g} .
\end{aligned}
$$

According to theorem of line of curvature, the parameter curves on surface are also lines of curvature if and only if $g_{12}=h_{12}=0$. Then $g_{12}=h_{12}=0$ implies that $\tau_{g}=0$ that means the curve center $c(u)$ is a line of curvature on $M$.

Theorem 4. Let $X(u, v)$ be a regular spacelike tubular surface with Darboux frame in Minkowski 3-space $\mathrm{E}_{1}^{3}$.

(i) If the center curve $c(u)$ is a geodesic on the surface $M$, then the Gaussian and mean curvature of tubular surface given by Eqn. (7) are as follows:

$$
\begin{aligned}
& K=\frac{\kappa \sinh v}{r(1+r \kappa \sinh v)} \\
& H=\frac{2 r \kappa \sinh v+1}{2 r(1+r \kappa \sinh v)} .
\end{aligned}
$$

(ii) If the center curve $c(u)$ is an asymptotic curve on the surface $M$, then the Gaussian and mean curvature of tubular surface given by Eqn. (7) are as follows:

$$
\begin{aligned}
& K=\frac{\kappa \cosh v}{r(r \kappa \cosh v-1)}, \\
& H=\frac{2 r \kappa \cosh v-1}{2 r(r \kappa \cosh v-1)} .
\end{aligned}
$$

Proof. From equations (4), (6), (15) and (16) we obtain

$$
\left\{\begin{array}{l}
g_{11}=\left(1-r \kappa_{g} \cosh v+r \kappa_{n} \sinh v\right)^{2}-r^{2} \tau_{g}^{2} \\
g_{22}=-r^{2}
\end{array}\right.
$$

and the Gaussian and mean curvature for $X(u, v)$ are

$$
\begin{aligned}
& K=\frac{\kappa_{n} \sinh v-\kappa_{g} \cosh v}{r\left[1+r\left(\kappa_{n} \sinh v-\kappa_{g} \cosh v\right)\right]} \\
& H=\frac{r\left(\kappa_{n} \sinh v-\kappa_{g} \cosh v\right)+1}{2 r\left[1+r\left(\kappa_{n} \sinh v-\kappa_{g} \cosh v\right)\right]} .
\end{aligned}
$$

respectively.

(i) Since the center curve $c(u)$ is a geodesic on the surface $M$. So, $\kappa_{g}=0, \kappa_{n}=\kappa$ and $\tau_{g}=\tau$. If we substitute these above then the Gaussian and mean curvature of tubular surface given by

$$
\begin{aligned}
& K=\frac{\kappa \sinh v}{r(1+r \kappa \sinh v)}, \\
& H=\frac{2 r \kappa \sinh v+1}{2 r(1+r \kappa \sinh v)} .
\end{aligned}
$$


(ii) Because the center curve $c(u)$ is an asymptotic curve on the surface $M$, then $\kappa_{n}=0, \kappa_{g}=\kappa$ and $\tau_{g}=\tau$. Then it gathers that

$$
\begin{aligned}
& K=\frac{\kappa \cosh v}{r(r \kappa \cosh v-1)}, \\
& H=\frac{2 r \kappa \cosh v-1}{2 r(r \kappa \cosh v-1)} .
\end{aligned}
$$

\section{References}

[1] M. Do Carmo, Differential Geometry of Curves and Surfaces, Prentice-Hall Inc., Englewood Cliffs, New Jersey, 1976.

[2] R.T. Farouki, C.A. Neff, Analytic properties of plane offset curves, Comput. Aided Geom. Design 7, (1990), 83-99.

[3] R.T. Farouki, C.A. Neff, Algebraic properties of plane offset curves, Comput. Aided Geom. Design 7, (1990), 101-127.

[4] R.T. Farouki, R. Sverrissor, Approximation of rolling-ball blends for free-form parametric surfaces, Comput.-Aided Design, 28, (1996), 871-878.

[5] T. Maekawa, M. N. Patrikalakis, T. Sakkalis and G. Yu, Analysis and applications of pipe surfaces, Computer Aided Geometric Design 15 (1998),437-458.

[6] B. O’Neill, Semi-Riemannian geometry with applications to relativity, Academic press, New York, 1983.

[7] U. Shani, D.H. Ballard, Splines as embeddings for generalized cylinders, Comput. Vision Graphics Image Process, 27, (1984), $129-156$.

[8] A. Uçum, K. İarslan, New Types of Canal Surfaces in Minkowski 3-Space, Adv. in Appl. Clifford Algeb., 26 (1), $2016,449-468$.

[9] L. Wang, M.C. Leu, D. Blackmore, Generating sweep solids for NC verification using the SEDE method, in: Proceedings of the Fourth Symposium on Solid Modeling and Applications, Atlanta, Georgia, 14-16 May 1997, pp. 364-375.

[10] F. Wang and H. Liu, Mannheim Partner Curves in 3-Space, Proceedings of The Eleventh International Workshop on Diff. Geom., 11 (2007), 25-31.

[11] Z. Xu, R. Feng and J. Sun, Analytic and algebraic properties of canal surfaces, J. of Comp. and Appl. Math., 195 (1), (2006) $220-228$. 